Classification

Physics Abstracts

43.35C $-46.30 \mathrm{C}-62.30 \mathrm{D}$

\title{
Evaluation de la matrice d'élasticité des composites orthotropes par propagation ultrasonore en dehors des plans principaux de symétrie
}

\author{
S. Baste et B. Hosten \\ Laboratoire de Mécanique Physique, U.R.A. C.N.R.S. n 867, Université de Bordeaux I, 351 Cours de la \\ Libération, 33405 Talence Cedex, France
}

(Reçu le 27 juin 1989, révisé le 7 septembre 1989 et le 27 octobre 1989, accepté le 2 novembre 1989)

\begin{abstract}
Résumé. - La caractérisation du comportement élastique de composites, possédant une symétrie orthotrope et se présentant sous forme de plaques minces, est réalisée à l'aide d'un spectro-interféromètre ultrasonore à immersion. L'identification complète des neuf composantes indépendantes du tenseur d'élasticité est effectuée à partir de mesures de vitesses de propagation dans les deux plans principaux de symétrie accessibles et dans un plan non principal. Dans ce plan, l'équation caractéristique du troisième degré ne se factorise pas ; trois ondes sont engendrées à l'interface liquide-solide.
\end{abstract}

\begin{abstract}
Elastic behaviour characterization of thin plate-shaped orthotropic composites are performed by an ultrasonic immersion spectro-interferometer. An identification process of the nine independent elastic stiffness constants is realized from the measurement of ultrasonic velocities in the two accessible principal planes and in a non-principal plane. In this plane, three modes are generated through the liquid-anisotropic solid interface and they are the eigenvalues of the third degree characteristic equation which, in this case, does not factorize.
\end{abstract}

\section{Introduction.}

La caractérisation des milieux anisotropes par des méthodes ultrasonores a fait l'objet de nombreux travaux. La détermination des constantes d'élasticité est basée sur la mesure des vitesses de propagation des différents modes se propageant dans des directions particulières du matériau. Une classification est faite usuellement par la nature de l'interaction transducteur-solide à évaluer. Les techniques à contact direct $[1,2]$, qui, comme les essais mécaniques classiques, nécessitent des coupes orientées suivant $n$ directions, $n$ croissant avec le degré d'anisotropie, ont été supplantées par des systèmes à immersion $[3,4]$. Ceux-ci offrent souvent des résultats plus reproductibles et autorisent de nombreuses mesures de célérités, dans des directions de propagation différentes pour un nombre réduit de coupes. C'est le cas, en particulier, du spectro-interféromètre $[5,6]$ qui est la base de notre instrumentation.

Ce banc à immersion utilise le principe de l'interféromètre numérique associée à un algorithme de poursuite des modes par une technique d'intercorré- lation. Les ondes de volumes quasi-longitudinales et quasi-transversales sont engendrées continûment en fonction de la direction de propagation par conversion de modes à l'interface liquide-solide. La conception du dispositif et l'utilisation d'une méthode d'optimisation [7] ont permis l'identification des constantes d'élasticité de multiples composites naturels ou artificiels $[8,9]$.

Toutefois, cette méthode, fondée sur des mesures des célérités dans des plans principaux de symétrie (i.e. plan contenant deux axes de symétrie), présente des lacunes pour certaines classes de symétrie. La caractérisation élastique complète des solides anisotropes possédant une symétrie d'ordre inférieur à la symétrie hexagonale, comme les composites à renforts 2D ou plus, exige au moins deux coupes orientées [8]. Produits uniquement en plaques minces, leur plan principal translaminaire n'est pas accessible aux mesures. Les raideurs manquantes n'autorisent pas l'inversion de la matrice d'élasticité et donc la comparaison avec les constantes de l'ingénieur issues d'essais mécaniques traditionnels. Lorsque le rapport épaisseur de l'échantil- 
lon/longueur d'onde devient trop petit, seules les ondes de plaques sont utilisables. Bien que récemment, le problème direct ait été en partie résolu $[10,11]$ dans les composites orthotropes, il n'a pas encore été montré que le problème inverse de la mesure de la matrice de raideur d'un composite à partir des courbes de dispersion ait une solution. Les ondes de surface sont une autre voie possible. Cependant, leur sensibilité à l'état de surface les rend peu représentatives des caractéristiques volumiques des matériaux. Elles nécessitent de toute façon plusieurs échantillons [12].

Les ondes de volume étant, sans doute, les mieux adaptées pour une évaluation qui intègre tout le volume d'un solide, nous présentons une extension de la technique du spectro-interféromètre à immersion. La détermination complète de la matrice d'élasticité d'un matériau orthotrope est réalisée à partir de mesures de célérités dans un plan non principal de la seule coupe orientée disponible.

Le problème théorique, que pose un tel dispositif, réside essentiellement dans la propagation dans une direction quelconque d'ondes de volume dans un milieu anisotrope et dans leur génération à l'interface liquide-solide. L'identification d'une partie du tenseur d'élasticité est menée en conservant deux plans de balayage angulaire particuliers que sont les plans principaux. L'évaluation élastique d'un matériau orthotrope est complétée par l'auscultation d'un plan non principal bien choisi. Le processus d'identification, basé sur la minimisation d'un système surdéterminé, dérivé des équations de propagation, est testé sur un composite carbone-époxy constitué d'une matrice époxy renforcée par un réseau unidirectionnel de fibres de carbone.

\section{Génération et propagation d'ondes de volume dans un solide anisotrope.}

L'étude de la propagation des ondes de volume dans un milieu anisotrope conduit à un problème classique aux valeurs propres. La résolution, par une méthode heuristique, de l'équation du mouvement linéarisée pour des petits déplacements $U$, sous la forme d'une onde plane homogène monochromatique non amortie :

$$
\mathbf{U}=a \mathbf{P} \exp (i \omega t-\mathbf{K} \cdot \mathbf{O M}),
$$

conduit aux équations de propagation [13] :

$$
\Gamma_{i \ell} P_{\ell}-\rho c^{2} P_{i}=0,
$$

dans lesquelles apparaissent l'amplitude de l'onde $a$, le vecteur de polarisation $\mathbf{P}$, la pulsation $\omega$, le temps $t$, le vecteur d'onde $\mathbf{K}$, le vecteur position OM, la masse volumique $\rho$, la vitesse de propagation $c$ et le tenseur de propagation $\Gamma_{i \ell}(i, \ell=1$ à 3$)$. Pour un solide anisotrope quelconque, ce tenseur s'écrit :

$$
\Gamma_{i \ell}=C_{i j k \ell} n_{j} n_{k}
$$

$n_{j}=c / \omega K_{j}$ sont les composantes du vecteur direction de propagation, exprimées dans un repère $\mathbf{X}_{i}(i=1$ à 3$)$ et $C_{i j k \ell}$ est le tenseur des rigidités.

Des considérations thermodynamiques montrent que ce tenseur est symétrique et que le nombre de ses composantes indépendantes varie de deux à vingt-et-un suivant le degré d'anisotropie [13]. Nous nous limitons au cas de la symétrie orthorhombique, qui est suffisamment générale pour décrire le comportement de la plupart des matériaux naturels ou non. En introduisant la notation contractée habituelle [13], le tenseur des rigidités à neuf composantes indépendantes est représenté par une matrice carrée $(6 \times 6)$ :

$$
\left[C_{I J}\right]=\left[\begin{array}{cccccc}
C_{11} & C_{12} & C_{13} & \mathrm{O} & \mathrm{O} & \mathrm{O} \\
C_{12} & C_{22} & C_{23} & \mathrm{O} & \mathrm{O} & \mathrm{O} \\
C_{13} & C_{23} & C_{33} & \mathrm{O} & \mathrm{O} & \mathrm{O} \\
\mathrm{O} & \mathrm{O} & \mathrm{O} & C_{44} & \mathrm{O} & \mathrm{O} \\
\mathrm{O} & \mathrm{O} & \mathrm{O} & \mathrm{O} & C_{55} & \mathrm{O} \\
\mathrm{O} & \mathrm{O} & \mathrm{O} & \mathrm{O} & \mathrm{O} & C_{66}
\end{array}\right]
$$

Les six composantes du tenseur de propagation s'écrivent [14] :

$$
\begin{aligned}
& \Gamma_{11}=C_{11} n_{1}^{2}+C_{66} n_{2}^{2}+C_{55} n_{3}^{2}, \\
& \Gamma_{12}=\left(C_{12}+C_{66}\right) n_{1} n_{2}, \\
& \Gamma_{13}=\left(C_{13}+C_{55}\right) n_{1} n_{3}, \\
& \Gamma_{23}=\left(C_{23}+C_{44}\right) n_{2} n_{3}, \\
& \Gamma_{22}=C_{66} n_{1}^{2}+C_{22} n_{2}^{2}+C_{44} n_{3}^{2}, \\
& \Gamma_{33}=C_{55} n_{1}^{2}+C_{44} n_{2}^{2}+C_{33} n_{3}^{2}, \\
& \Gamma_{21}=\Gamma_{12}, \Gamma_{31}=\Gamma_{13}, \Gamma_{32}=\Gamma_{23} .
\end{aligned}
$$

Les célérités et les polarisations des ondes planes se propageant suivant une direction quelconque $\mathbf{n}=\left(n_{1}, n_{2}, n_{3}\right)$ dans un solide orthotrope de rigidité $C_{I J}(I, J=1$ à 6$)$, s'obtiennent en cherchant les valeurs propres et les vecteurs propres du tenseur $\Gamma_{i \ell}$, solutions de l'équation caractéristique du troisième degré [13]:

$$
\left|\Gamma_{i \ell}-\rho c^{2} \delta_{i \ell}\right|=0,
$$

exprimant la condition de compatibilité des trois équations homogènes (2).

Comme le tensur $\Gamma_{i \ell}$ est symétrique, ses valeurs propres sont réelles et positives et ses vecteurs propres orthogonaux. Il existe, dans le cas général, trois ondes planes susceptibles de se propager dans une même direction avec des célérités différentes, les polarisations étant orthogonales [14]. Dans un milieu anisotrope, excepté dans des directions particulières, il n'existe pas de mode pur (longitudinal ou transversal). L'onde dont le vecteur polarisation est le plus proche du vecteur d'onde $\mathbf{K}$ est appelée onde quasi-longitudinale (QL), les deux autres sont appelées quasi-transversales (QT1) et QT2). 
La génération de ces trois modes est réalisée, dans un dispositif à immersion par réfraction à l'interface liquide-solide anisotrope ; c'est un problème à cinq ondes définies par leur nombre d'ondes $k_{m}$ et leur direction de propagation $\mathbf{n}_{m}$ : l'onde incidente dans le liquide, longitudinale pure $\left(k_{\mathrm{e}}, \mathbf{n}_{\mathrm{e}}\right)$, l'onde longitudinale réfléchie $\left(k_{\mathrm{r}}, \mathbf{n}_{\mathrm{r}}\right)$ et les trois modes transmis dans le solide $\left(k_{1}, \mathbf{n}_{1}\right),\left(k_{2}, \mathbf{n}_{2}\right)$ et $\left(k_{3}, \mathbf{n}_{3}\right)$ reliés par les lois de Snell-Descartes :

$$
\begin{array}{r}
k_{\mathrm{c}} \sin \left(\mathbf{x}_{1}, \mathbf{n}_{\mathrm{e}}\right)=k_{\mathrm{r}} \sin \left(\mathbf{x}_{1}, \mathbf{n}_{\mathrm{r}}\right)=k_{1} \sin \left(\mathbf{x}_{1}, \mathbf{n}_{1}\right)= \\
=k_{2} \sin \left(\mathbf{x}_{1}, \mathbf{n}_{2}\right)=k_{3} \sin \left(\mathbf{x}_{1}, \mathbf{n}_{3}\right),
\end{array}
$$

où la direction $\mathbf{x}_{1}$ est choisie comme la normale à l'interface.

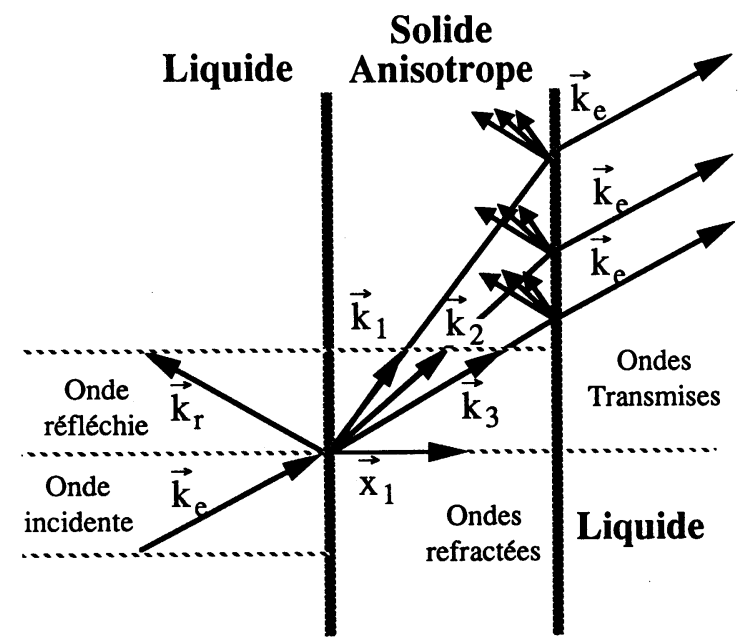

Fig. 1. - Génération de trois modes à l'interface liquide/solide.

[Three modes generation through liquid/solid interface.]

Le nombre des ondes effectivement engendrées à l'interface est précisé par la résolution des équations de continuité à l'interface de la vitesse et des contraintes normales.

\section{Propagation dans les plans principaux.}

L'équation caractéristique (6) ne peut être résolue analytiquement, d'une manière simple, que pour des plans particuliers pour lesquels au moins deux des trois composantes non diagonales du tenseur de propagation sont nulles. C'est précisément la propriété des trois plans principaux que possèdent les matériaux à symétrie orthotrope (Fig. 2). L'étude de la propagation dans ces plans fournit les neuf constantes élastiques indépendantes mais plusieurs coupes orientées sont nécessaires [8]. La seule coupe disponible dans les composites produits en plaques minces de quelques millimètres d'épaisseur, rend possible uniquement l'auscultation des plans principaux $\left(\mathbf{x}_{1}, \mathbf{x}_{2}\right)$ et $\left(\mathbf{x}_{1}, \mathbf{x}_{3}\right)$. Ces deux plans de balayage particuliers autorisent l'identification d'une partie de la matrice d'élasticité.

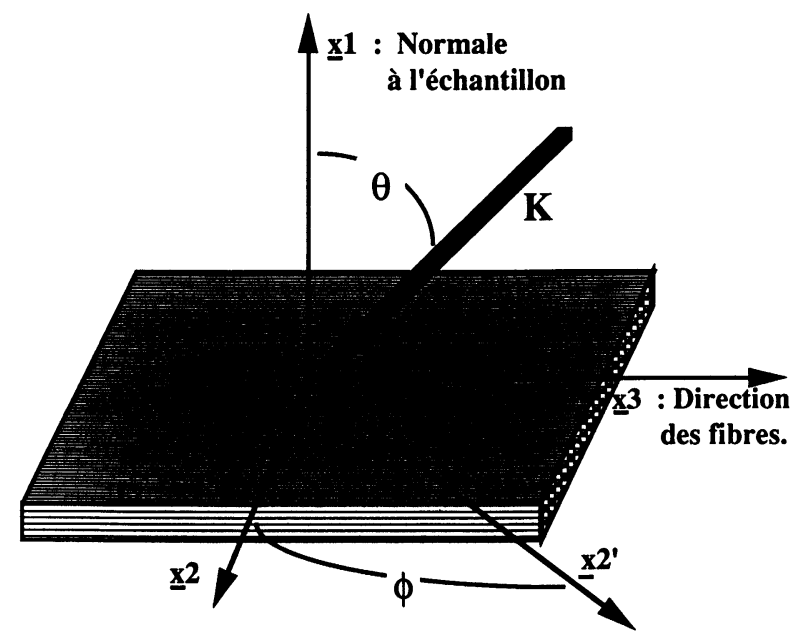

Fig. 2. - Définition du repère principal lié au matériau et du repère de propagation. L'échantillon est ausculté suivant un plan $\phi=$ Cte (plan $\mathbf{x}_{1}, \mathbf{x}_{2}^{\prime}$ ).

Suivant les lois de Snell/Descartes, ce plan constitue le plan d'incidence et le plan de propagation pour tous les modes. $\phi=0^{\circ}$ et $\phi=90^{\circ}$ constituent deux plans principaux.

[Definition of principal planes in the material and of propagation plane.

Sample is scanned following the $\phi=$ Cte plane. According Snell's laws, it is the incidence and propagation plane for all modes. $\phi=0^{\circ}$ and $\phi=90^{\circ}$ are two principal planes.]

Nous étudions d'abord la propagation dans le plan $\left(x_{1}, x_{2}\right)$, la démarche étant identique pour l'autre plan principal $\left(\mathrm{x}_{1}, \mathrm{x}_{3}\right)$. Dans ce plan, $n_{3}=0$ et les éléments $\Gamma_{13}$ et $\Gamma_{23}$ du tenseur de propagation sont nuls, l'équation caractéristique (6) développée se factorise [15] :

$$
\begin{aligned}
f_{k}\left(C_{I J}\right)=\left[\Gamma_{33}-\lambda\right]\left[\lambda^{2}-\lambda(\right. & \left.\Gamma_{11}+\Gamma_{22}\right)+ \\
& \left.+\Gamma_{11} \Gamma_{22}-\Gamma_{12}^{2}\right]
\end{aligned}
$$

où $\lambda=\rho c^{2}$ sont les valeurs propres $\mathrm{du}$ tenseur $\Gamma_{i \ell}$. La valeur propre triviale $\rho c^{2}=\Gamma_{33}$ correspond à une onde transversale pure polarisée suivant la direction $x_{3}$. Les conditions aux limites, $u_{3}=0$, à l'interface liquide-solide, montrent que ce mode n'est pas engendré sous incidence oblique dans ce plan principal. Les célérités des deux modes générés, une onde quasi-longitudinale et une onde quasitransversale polarisées dans le plan $\left(\mathrm{x}_{1}, \mathrm{x}_{2}\right)$ orthogonal à la direction propre $x_{3}$, sont solutions de l'équation du second degré (8) et vérifient l'expression analytique [13] :

$$
2 \rho c^{2}-\left(\Gamma_{11}+\Gamma_{22}\right) \pm \sqrt{\left(\Gamma_{11}-\Gamma_{22}\right)^{2}+4 \Gamma_{12}^{2}},
$$

le signe $(+$ ou -$)$ dépend de la polarisation des deux modes.

La procédure d'identification, au sens des moin- 
dres carrés, détermine à partir de l'expression analytique des célérités (9) et de l'ensemble de leurs mesures expérimentales dans ce plan principal, les constantes d'élasticité qui influencent la propagation des deux modes générés [7]. Les relations (5) et $n_{3}=0$ montrent qu'elles sont au nombre de quatre : $C_{11}, C_{22}, C_{66}$ et $C_{12}$.

Dans le plan $\left(x_{1}, x_{2}\right), n_{2}=0$, l'onde transversale pure qui correspond à la valeur propre triviale $\rho c^{2}=\Gamma_{22}$ n'est pas non plus engendrée à l'interface.

L'identification dans ce plan principal donne trois autres raideurs à partir de l'équation (9) en effectuant les substitutions suivantes :

$$
C_{22} \rightarrow C_{33}, \quad C_{12} \rightarrow C_{13}, \quad C_{66} \rightarrow C_{55} .
$$

\section{Acquisition des vecteurs d'ondes.}

Les vecteurs d'ondes dans un plan contenant la normale à l'échantillon (Fig. 2) sont mesurés à l'aide d'un banc ultrasonore de caractérisation des matériaux composites dont la description détaillée est donnée dans des travaux antérieurs [5-9]. Rappelons ici brièvement que l'échantillon sous-test est immergé dans l'eau et placé entre deux transducteurs ultrasonores à large bande et de fréquence centrale de l'ordre de $5 \mathrm{MHz}$. Un goniomètre fait varier continûment l'angle $\theta$ (Fig. 2) entre la direction de l'onde incidente et la normale à l'échantillon. Le premier interface eau/solide (Fig. 1) réalise la conversion du mode longitudinal incident en modes quasi-longitudinal et/ou quasi-transversal dans le solide, puis le second interface la conversion inverse. La mesure précise des célérités en fonction de la direction de propagation est effectuée par un logiciel de séparation et de poursuite des modes [5].

L'échantillon se présente sous forme d'une plaque de dimensions $50 \times 50 \times 3,4 \mathrm{~mm}$. Les équations de propagation (2) sont établies pour un milieu homogène et infini, il existe donc, des conditions sur la fréquence des ondes, en relation avec la structure du matériau, et les dimensions de l'échantillon. Les conditions de milieu infini sont satisfaites si d'une part l'encombrement spatial du train d'ondes ultrasonores est réduit et d'autre part l'épaisseur est suffisante pour assurer la séparation des modes. Les effets du mélange des modes sur ces mesures ont été analysés [15] et l'épaisseur de cet échantillon est nettement supérieure à la limite inférieure pour laquelle les réflexions internes dans l'échantillon faussent les mesures [16].

Les conditions d'homogénéité sont satisfaites si la longueur d'onde est plus grande que les dimensions des inhomogénéités dans le matériau. Les matériaux composites unidirectionnels à fibres longues sont des matériaux tests pour cette méthode ultrasonore en raison de la très grande anisotropie de leurs propriétés mécaniques. Les résultats présentés ici sont relatifs à un composite 1D à matrice époxy et fibres de carbone. Le diamètre des fibres, 7 à 8 microns, est très petit devant les longueurs d'ondes voisines de 0,5 mm. Ces matériaux sont fabriqués par empilement de plis. L'échantillon, de masse volumique $\rho=1,5 \mathrm{~kg} / \mathrm{m}^{3}$, possède trente plis, l'épaisseur d'un pli est de l'ordre de $0,1 \mathrm{~mm}$. Les conditions d'homogénéité restent parfaitement vérifiées car les conditions de cuisson (température et pression) imposent aux plis de s'interpénétrer. Ainsi l'interface inter-

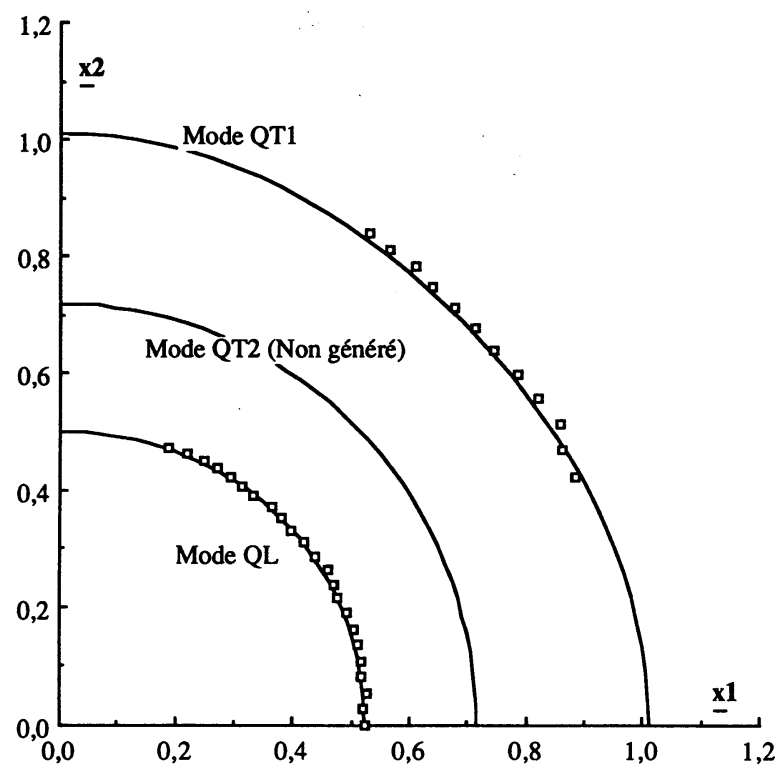

a)

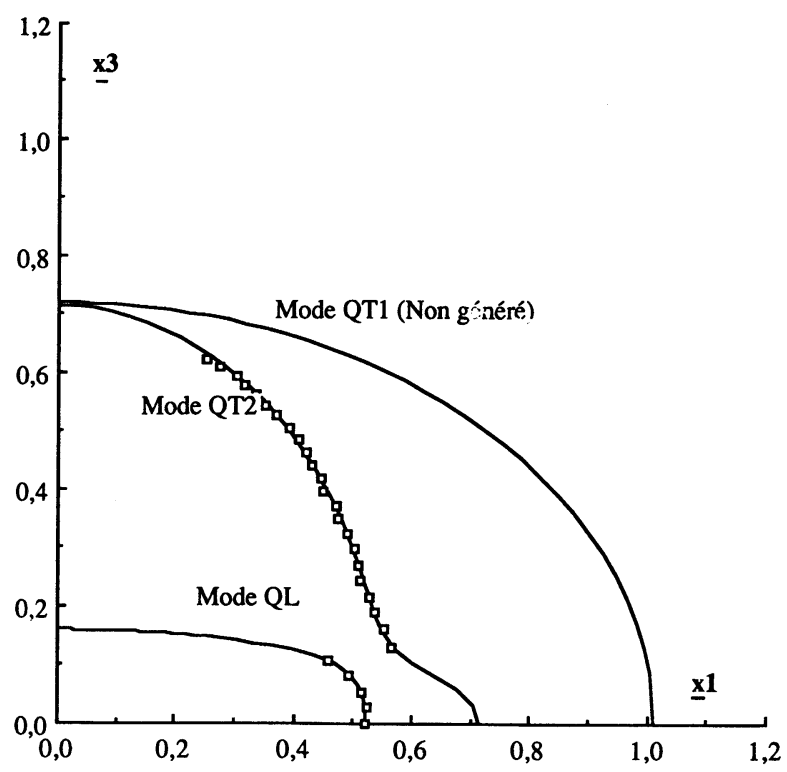

b)

Fig. 3. - Courbes des lenteurs normalisées a) dans le plan $\left(\mathbf{x}_{1}, \mathbf{x}_{2}\right)$, b) dans le plan $\left(\mathbf{x}_{1}, \mathbf{x}_{3}\right)$.

[Normalized slowness curves a) in $\left(\mathbf{x}_{1}, \mathbf{x}_{2}\right)$ plane, b) in $\left(\mathbf{x}_{1}, \mathbf{x}_{3}\right)$ plane. 
plis est excessivement réduit et il n'y a pas de réflexion interne dans le matériau. Ce mode de fabrication est la raison principale de la symétrie orthotrope du matériau, étudiée au paragraphe 5 .

Les courbes de lenteurs dans les plans principaux sont présentées sur la figure 3. Elles sont normalisées par le nombre d'onde $k_{\mathrm{e}}\left(c_{\mathrm{e}}=1496 \mathrm{~m} / \mathrm{s}\right.$ à $\left.20^{\circ} \mathrm{C}\right)$ dans l'eau. Les faibles écarts quadratiques $(0,66 \%$ pour le plan $\left(x_{1}, x_{2}\right)$ et $0,58 \%$ pour le plan $\left.\left(x_{1}, x_{3}\right)\right)$ entre les données expérimentales ( $\square$ ) et calculées (lignes continues) confirment la qualité des valeurs identifiées des constantes d'élasticité :

$$
\left[C_{I J}\right]=\left[\begin{array}{cccccc}
12 & 5,6 & 4,9 & & & \\
5,6 & 13 & - & \mathrm{GPa} & & \\
4,9 & - & 127 & & & \\
& & & - & & \\
& & & & 6,4 & \\
& & & & & 3,2
\end{array}\right]
$$

Dans le plan $\left(\mathbf{x}_{1}, \mathbf{x}_{2}\right)$, la valeur du facteur d'anisotropie, $2 C_{66} /\left(C_{22}-C_{12}\right)=0,98$, est très proche de l'isotropie $\left(2 C_{66} /\left(C_{22}-C_{12}\right)=1\right)$. Se pose le problème du choix, a priori, de la symétrie du matériau. L'observation visuelle de la texture du matériau. n'est pas suffisante.

L'échantillon carbone-époxy à fibres longues assez bien orientées autour de l'axe principal $x_{3}$ peut présenter plusieurs symétries suivant la distribution géométrique des fibres $[6,9]$. Si la symétrie hexagonale à cinq constantes indépendantes est, a priori, une bonne représentation de l'anisotropie de ce composite, une analyse plus fine montre qu'elle n'est pas exacte puisque $C_{11}$ est légèrement différent de $C_{22}$ et que le plan $\left(\mathbf{x}_{1}, \mathbf{x}_{2}\right)$ n'est pas rigoureusement isotrope. Par conséquent; les égalités entre $C_{13}$ et $C_{23}$ d'une part et $C_{44}$ et $C_{55}$ d'autre part, caractéristiques d'une symétrie hexagonale d'axe sénaire $\mathbf{x}_{3}$, ne sont pas parfaitement vérifiées. Les deux constantes $C_{23}$ et $C_{44}$ restent à déterminer pour compléter la matrice d'élasticité.

\section{Propagation hors plans principaux.}

Pour un plan non principal, l'équation caractéristique (6) associée au tenseur de propagation ne se factorise pas. Par conséquent, les valeurs analytiques des valeurs propres du tenseur $\Gamma_{i \ell}$ ne sont plus disponibles. Il devient nécessaire, pour obtenir l'expression des vitesses de phase, en fonction des constantes d'élasticité, de résoudre l'équation de propagation (6), du troisième degré en $\rho c^{2}$, soit sous forme développée [18] :

$$
\begin{aligned}
\left(\Gamma_{11}-\rho c^{2}\right)[ & \left.\left(\Gamma_{22}-\rho c^{2}\right)\left(\Gamma_{33}-\rho c^{2}\right)-\Gamma_{23}^{2}\right]- \\
& -\Gamma_{13}^{2}\left(\Gamma_{22}-\rho c^{2}\right)-\Gamma_{12}^{2}\left(\Gamma_{33}-\rho c^{2}\right) \\
& +2 \Gamma_{12} \Gamma_{13} \Gamma_{23}=0 .
\end{aligned}
$$

Pour un solide anisotrope, les relations (5) mon- trent qu'aucune composante $\Gamma_{i \ell}$ du tenseur de propagation dans un plan non principal n'est nulle. Dès lors, il n'existe pas de valeur propre triviale ; aucune direction de propagation n'est confondue avec les axes $\mathbf{x}_{i}$.

Considérons maintenant le problème de réflexionréfraction décrit par la figure 1. Le plan d'incidence est défini par la normale à l'échantillon $x_{1}$ et un axe $\mathbf{x}_{2}^{\prime}$ situé dans le plan d'interface tel que l'angle $\left(x_{2}, x_{2}^{\prime}\right)=\phi$ (Fig. 2). Les composantes du vecteur direction de propagation dans le plan d'incidence s'expriment par :

$$
\begin{aligned}
& n_{1}^{\mathrm{e}}=\cos \theta, \\
& n_{2}^{\mathrm{e}}=\sin \theta \cos \phi, \\
& n_{3}^{\mathrm{e}}=\sin \theta \sin \phi,
\end{aligned}
$$

$\theta$ étant l'angle d'incidence par rapport à la normale à l'échantillon $\mathbf{x}_{1}$.

Les relations (7) entre l'onde incidente dans le liquide $\left(k_{\mathrm{e}}, \mathbf{n}_{\mathrm{e}}\right)$, l'onde réfléchie $\left(k_{\mathrm{r}}, \mathbf{n}_{\mathrm{r}}\right)$ et les ondes réfractées $\left(k_{i}, \mathbf{n}_{i}\right), i=1$ à 3 , et la continuité des contraintes et des déplacements à l'interface,

$$
\begin{aligned}
\mathbf{u}^{\mathrm{c}} \cdot \mathbf{x}_{1}+\left.\mathbf{u}^{\mathrm{r}} \cdot \mathbf{x}_{1}\right|_{x_{1}=0} & =\mathbf{u}^{1} \cdot \mathbf{x}_{1}+\mathbf{u}^{2} \cdot \mathbf{x}_{1}+\left.\mathbf{u}^{3} \cdot \mathbf{x}_{1}\right|_{x_{1}=0} \\
\sigma_{i j}^{\mathrm{c}} \cdot \mathbf{x}_{1}+\left.\sigma_{i j}^{\mathrm{r}} \cdot \mathbf{x}_{1}\right|_{x_{1}=0} & =\sigma_{i j}^{1} \cdot \mathbf{x}_{1}+ \\
& +\sigma_{i j}^{2} \cdot \mathbf{x}_{1}+\left.\sigma_{i j}^{3} \cdot \mathbf{x}_{1}\right|_{x_{1}=0}
\end{aligned}
$$

où $\sigma_{i j}^{m}, i, j=1$ à 3 , est le tenseur des contraintes associé à l'onde $m$, procurent le système d'équations algébriques (12), autorisant le calcul des amplitudes des modes réfléchis/réfractés à l'interface liquidesolide anisotrope. La résolution de ce système de Cramer de quatre d'équations permet de préciser les conditions de génération des modes dans un plan non principal d'un solide anisotrope $[19,20]$.

La constante d'élasticité $C_{23}$ n'apparaît que dans une seule composante non diagonale du tenseur de propagation $\Gamma_{23}$. D'après les relations (5) et (11),

$$
\Gamma_{23}=\left(C_{23}+C_{44}\right) \sin ^{2} \theta \cos \phi \sin \phi,
$$

l'influence de $C_{23}$ sur les vitesses de propagation est maximale pour un angle $\phi=45^{\circ}$. Il assure l'ouverture angulaire la plus grande avec l'auscultation des plans principaux $\left(\mathbf{x}_{1}, \mathbf{x}_{2}\right)$ et $\left(\mathbf{x}_{1}, \mathbf{x}_{3}\right)$.

L'acquisition des lenteurs (Fig. 4) et des amplitudes transmises par l'échantillon (Fig. 5) est effectuée dans ce plan non principal, en fonction de l'angle d'incidence $\theta$, en poursuivant les trois modes ; le mode quasi-longitudinal $\mathbf{Q L}$ et les deux modes quasi-transversaux QT1 et QT2.

\section{Identification des constantes d'élasticité.}

La totalité des relevés expérimentaux de célérité des trois modes est solution de l'ensemble des équations 


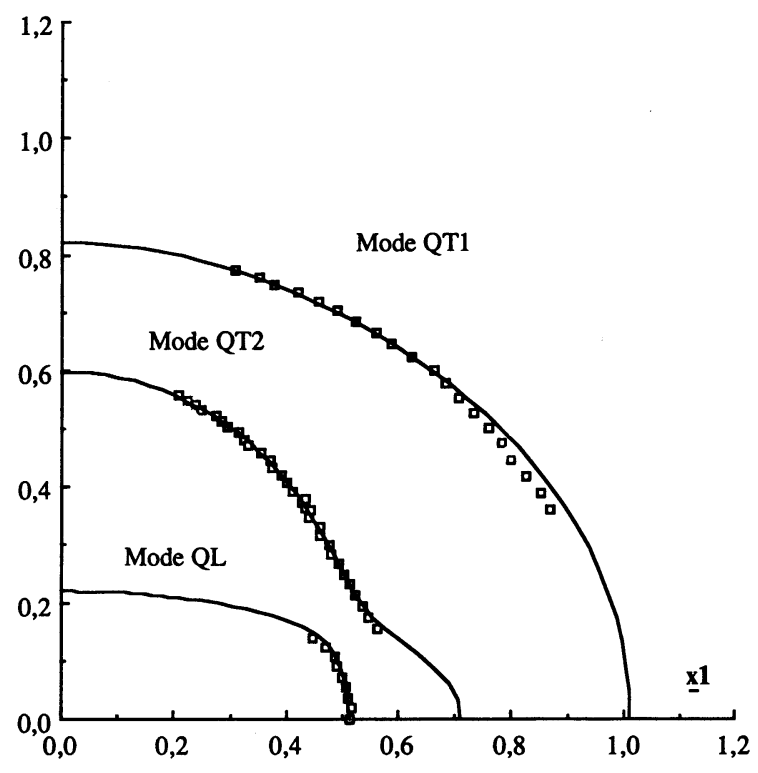

Fig. 4. - Courbes des lenteurs normalisées dans le plan $\phi=45^{\circ}$.

[Normalized slowness curves in $\phi=45^{\circ}$ plane.]

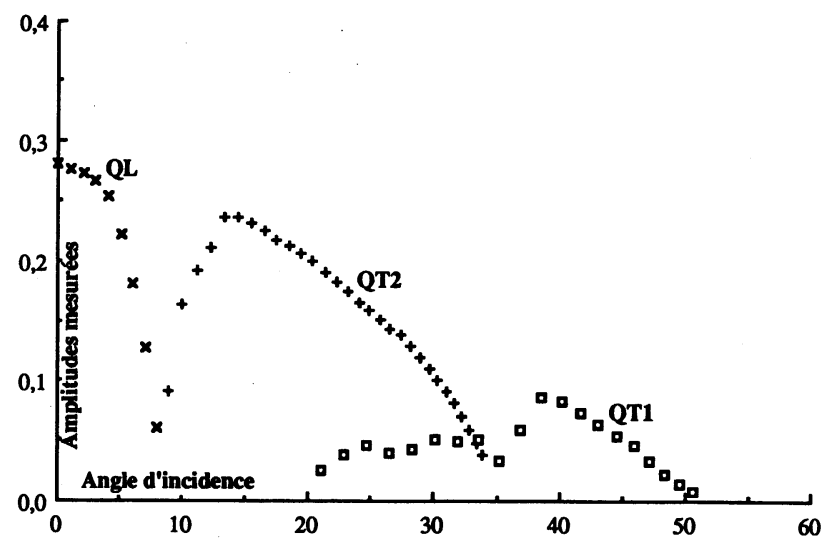

Fig. 5. - Amplitudes transmises par l'échantillon pour les trois modes générés dans le solide en fonction de l'angle d'incidence $\theta$ dans le plan $\phi=45^{\circ}$.

[Transmitted amplitudes through the sample for the three modes, versus incidence angle $\theta$ in the $\phi=45^{\circ}$ plane.]

cubiques non linéaires, déduites de l'équation caractéristique (10), aux perturbations expérimentales près [17] :

$$
\begin{aligned}
f_{k}\left(C_{I J}\right)=-\lambda^{3}+\lambda^{2}\left[\Gamma_{11}+\Gamma_{22}\right. & \left.+\Gamma_{33}\right]+\lambda\left[\Gamma_{12}^{2}+\Gamma_{13}^{2}+\Gamma_{23}^{2}-\Gamma_{11} \Gamma_{22}-\Gamma_{11} \Gamma_{33}-\Gamma_{22} \Gamma_{33}\right]+ \\
& +\Gamma_{11} \Gamma_{22} \Gamma_{33}+2 \Gamma_{12} \Gamma_{13} \Gamma_{23}-\Gamma_{11} \Gamma_{23}^{2}-\Gamma_{22} \Gamma_{13}^{2}-\Gamma_{33} \Gamma_{12}^{2} \approx 0,
\end{aligned}
$$

dans lesquelles $k \in[1, N], N$ étant le nombre total de mesures.

L'identification des constantes d'élasticité, au sens des moindres carrés, à partir de mesures de célérités revient à minimiser la fonctionnelle euclidienne, dérivée du système surdéterminé d'équations (14) [17] :

$$
F\left(C_{I J}\right)=\sum_{k=1}^{N}\left\{f_{k}\left(C_{I J}\right)\right\}^{2},
$$

par une méthode itérative de type Newton mettant en cuvre son gradient et sa matrice hessienne [21].

L'auscultation des deux plans principaux $\left(\mathbf{x}_{1}, \mathbf{x}_{2}\right)$ et $\left(\mathbf{x}_{1}, \mathbf{x}_{3}\right)$ donne accès à sept des neuf constantes d'élasticité. Ainsi, d'excellentes valeurs initiales sont disponibles pour la minimisation de la fonctionnelle (15). La diminution du nombre d'inconnues, de neuf à deux : $C_{23}$ et $C_{44}$ simplifie l'exigence d'une méthode de Newton vis-à-vis de l'initialisation [21] et favorise la convergence de l'algorithme.

Le gradient et la matrice hessienne de la fonctionnelle euclidienne s'écrivent, en fonction des inconnues à identifier, $X_{1}=C_{23}$ et $X_{2}=C_{44}$ :

$$
\begin{gathered}
\frac{\partial F}{\partial X_{i}}=\sum_{k=1}^{N} 2 \frac{\partial f_{k}}{\partial X_{i}} f_{k}, \\
\frac{\partial^{2} F}{\partial X_{i} \partial X_{j}}=\sum_{k=1}^{N}\left\{2 \frac{\partial f_{k}}{\partial X_{i}} \frac{\partial f_{k}}{\partial X_{j}}+2 f_{k} \frac{\partial^{2} f_{k}}{\partial X_{i} \partial X_{j}}\right\},
\end{gathered}
$$

avec

$$
\begin{gathered}
\frac{\partial f_{k}}{\partial X_{1}}=n_{2} n_{3}\left(2 \lambda \Gamma_{23}+2 \Gamma_{12} \Gamma_{13}-2 \Gamma_{11} \Gamma_{23}\right) \\
\frac{\partial f_{k}}{\partial X_{2}}=n_{3}^{2}\left(\lambda^{2}-\lambda\left(\Gamma_{11}+\Gamma_{33}\right)+\Gamma_{11} \Gamma_{33}-\Gamma_{13}^{2}\right)+ \\
+n_{2}^{2}\left(\lambda^{2}-\lambda\left(\Gamma_{11}+\Gamma_{22}\right)+\Gamma_{11} \Gamma_{22}-\Gamma_{12}^{2}\right) \\
+n_{2} n_{3}\left(2 \lambda \Gamma_{23}+2 \Gamma_{12} \Gamma_{13}-2 \Gamma_{11} \Gamma_{23}\right), \\
\frac{\partial^{2} f_{k}}{\partial X_{1}^{2}}=n_{2}^{2} n_{3}^{2}\left(2 \lambda-2 \Gamma_{11}\right), \frac{\partial^{2} f_{k}}{\partial X_{2}^{2}}=0 \\
\frac{\partial^{2} f_{k}}{\partial X_{1} \partial X_{2}}=n_{2}^{2} n_{3}^{2}\left(2 \lambda-2 \Gamma_{11}\right)
\end{gathered}
$$


Les résultats obtenus à partir de l'ensemble des célérités du plan (Fig. 4) $\phi=45^{\circ}$ complètent la matrice des raideurs :

$$
C_{23}=4,0 \mathrm{GPa} \text { et } C_{44}=6,3 \mathrm{GPa} .
$$

Les courbes en trait plein de la figure 4 sont les lenteurs recalculées dans le plan $\phi=45^{\circ}$ à l'aide des valeurs identifiées des constantes d'élasticité :

$$
\left[C_{I J}\right]=\left[\begin{array}{cccccc}
12 & 5,6 & 4,9 & & & \\
& 13 & 4,0 & & \text { GPa } & \\
& & 127 & & & \\
& & & 6,3 & & \\
& \text { Sym. } & & & 6,4 & \\
& & & & & 3,2
\end{array}\right] \text {. }
$$

L'écart très faible entre les données expérimentales ( $\square$ ) et calculées pour les trois modes générés, montre que cette matrice est une bonne représentation du comportement élastique du matériau. Cette matrice, à neuf constantes indépendantes, caractéristique d'un système de symétrie orthorhombique, montre que, dans un composite unidirectionnel, la distribution des fibres n'est probablement pas parfaitement aléatoire dans le plan perpendiculaire à l'axe $x_{3}$. Le choix d'une symétrie hexagonale n'est qu'une approximation de la représentation de l'anisotropie de ce composite carbone-époxy. La connaissance de toutes les composantes de la matrice de rigidité rend maintenant possible la détermination des constantes de l'ingénieur via son inversion.

\section{Conclusion.}

Le système de symétrie orthorhombique est suffisamment général pour décrire l'anisotropie de la plupart des composites de synthèse. La faible épaisseur $(2$ à $3 \mathrm{~mm})$ des pièces industrielles réalisées en composite rend impossible leur caractérisation élastique par des méthodes d'évaluation mécanique ou acoustique nécessitant plusieurs coupes orientées. En possession d'une seule coupe, la détermination complète des neuf constantes d'élasticité indépendantes n'est faisable qu'à travers la génération et l'analyse des ondes de volume se propageant dans des plans non principaux.

L'identification des constantes d'élasticité est réalisée par minimisation d'une fonctionnelle euclidienne cubique, dérivée de l'équation caractéristique de propagation. La totalité des mesures précises des célérités des trois modes, l'onde quasi-longitudinale et les deux ondes quasi-transversales, générées sous incidence oblique à l'interface liquide-solide, réalisées à l'aide d'un spectro-interféromètre numérique à immersion, sont prises en compte par cette fonctionnelle.

Les résultats obtenus, pour un carbone-époxy unidirectionnel, souligne le caractère hasardeux du choix, a priori d'une classe de symétrie de degré élevé comme est la symétrie hexagonale. En toute rigueur, l'anisotropie de ce composite doit être représentée par le système orthorhombique.

L'évaluation élastique complète des solides anisotropes présentant cette symétrie ouvre le domaine d'application des méthodes ultrasonores à de nombreuses textures; les composites $2 \mathrm{D}$ renforcés par des empilements de couche de fibre monodirectionnelle, $0^{\circ}-90^{\circ}$ par exemple, ou de tissus croisés ou bien encore les substrats plus complexes tels que les renforcements $3 \mathrm{D}$, pour lesquels la comparaison avec les constantes de l'ingénieur, déduites d'essais mécaniques conventionnels, est désormais permise. La prise en compte de l'amortissement anisotrope du milieu reste un problème à résoudre en introduisant la notion d'ondes hétérogènes [22].

Toutefois, cette procédure d'identification des constantes d'élasticité a été mise en échec pour quelques composites 2D industriels. L'examen des mesures réalisées dans leurs plans réputés principaux révèle la présence du troisième mode propre à une propagation hors plan principal. Les deux postulats de cette procédure d'évaluation: l'existence des plans principaux de symétrie et la coïncidence des axes principaux et des axes géométriques de l'échantillon ne sont pas satisfaits, ce qui peut expliquer cet insuccès.

La recherche des axes principaux et la mesure des parallaxes angulaires (angle d'Euler) dans l'alignement des axes principaux et des axes géométriques ont été abordées récemment pour la symétrie hexagonale et dans le cas d'un seul parallaxe angulaire [18]. L'extension au système orthorhombique incluant les trois angles d'Euler demande simplement un effort supplémentaire de calcul. De même, le prolongement de cette méthode d'évaluation élastique, utilisant des mesures de propagation hors plan principal, aux classes d'anisotropie plus marquée ne possédant plus de plan principaux, comme les systèmes monocliniques ou tricliniques, augmente seulement, aux problèmes de convergence près, le nombre des constantes à identifier. 


\section{Bibliographie}

[1] PAPAdakis E. P., Ultrasonic attenuation and velocity in three transformation products in steel, $J$. Appl. Phys. 35 (1964) 1474-1782.

[2] PAPADAKIS E. P., Ultrasonic phase velocity by pulseecho-overlap method incorporating diffraction phase corrections, J. Acoust. Soc. Am. 42 (1967) 1045-1051.

[3] Markham M. F., Measurement of the elastic constants of fibre composites by ultrasonics, Composites (mars 1970).

[4] SMITH R. E., Ultrasonic elastic constants of carbon fibers and their composites, J. Appl. Phys. 43 (1972) 2555-2561.

[5] Hosten B., BARrot A., Roux J., Interférométrie numérique ultrasonore pour la détermination de la matrice de raideur des matériaux composites, Acustica 53 (1983) 212-217.

[6] Roux J., Hosten B., Castagnede B., Deschamps M., Caractérisation mécanique des solides par spectro-interférométrie ultrasonore, Revue Phys. Appl. 20 (1985) 351-358.

[7] Hosten B., Castagnede B., Optimisation des constantes élastiques à partir des mesures de vitesses d'une onde ultrasonore, C.R. Acad. Sci. Paris Sér. II, 296 (1983) 297-300.

[8] Hosten B., Castagnede B., Mesures des constantes élastiques du bois à l'aide d'un interféromètre ultrasonore numérique et leur optimisation, C.R. Acad. Sci. Paris Sér. II, 296 (1983) 17611764.

[9] Castagnede B., Mesure des constantes élastiques de solides anisotropes par une méthode ultrasonore. Thèse $3^{\mathrm{e}}$ cycle, Bordeaux I (1984).

[10] Nayfeh A. H., Chimenti D. E., Ultrasonic wave reflection from liquid-coupled orthotropic plates with application to fibrous composites, J. Appl. Mech. 55 (1988) 863-870.

[11] Datta S. K., Shah A. H., Bratton R. L., ChaKRABORTY T., Wave propagation in laminated composite plates, J. Acous. soc. Am. 83 (1988) 2020-2026.
[12] BuCUR V., Rocaboy F., Surface wave propagation in wood : prospective method for the determination of wood off-diagonal terms of stiffness matrix, Ultrasonics 26 (1988) 344-347.

[13] Auld B. A., Acoustic fields and waves in solids (Wiley-Interscience, New York) Vol. 1 (1973).

[14] Dieulesaint E., Royer D., Ondes élastiques dans les solides (Masson, Paris) 1974.

[15] Castagnede B., Roux J., Hosten B., Correlation method for normal mode tracking in anisotropic media using an ultrasonic immersion system, Ultrasonics 27 (1989) 280-287.

[16] Hosten B., Anisotropic Materials Characterization by Simple or Double Transmission Ultrasonic Methods, Proceeding of Ultrasonics International 89, Madrid, 4/7 juillet 1989.

[17] BASTE S., DesChAMPS M., Identification des constantes d'élasticité à partir des vitesses de propagation dans un plan principal de symétrie, C.R. Acad. Sci. Paris Ser. II 309 (1989) 1521-1526.

[18] Castagnede B. et Sachse W., Optimized determination of elastic constants of anisotropic solids from wavespeed measurements, Rev. prog. $Q N D E$, Eds. D. O. Thompson and D. E. Chimenti (Plenum Press, New York) 8b (1989) 1855-1862.

[19] Rokhlin S. I., Bolland T. K., Adler L., Reflection and refraction of elastic waves on a plane interface between two generally anisotropic media, J. Acoust. Soc. Am. 79 (1986) 906-918.

[20] Ankan O., Telatar E., Atalar A., Reflection coefficient null of acoustic waves at a liquidanisotropic-solid interface, J. Acoust. Soc. am. 85 (1989) 1-10.

[21] Ciarlet P. G., Introduction à l'analyse numérique matricielle et à l'optimisation (Masson, Paris) 1982.

[22] Hosten B., Deschamps M., Tittman B. R., Inhomogeneous wave generation and propagation in lossy anisotropic solids. Application to the viscoelastic characterization of composite materials, J. Acoust. Soc. Am. 82 (1987) 1763-1770. 